\title{
A different detection method reveals a new role of alanine aminotransferase as an indicator of liver fibrosis
}

\author{
Pil Soo Sung, ${ }^{1,2}$
}

${ }^{1}$ Department of Internal Medicine and ${ }^{2}$ The Catholic University Liver Research Center, College of Medicine, The Catholic University of Korea, Seoul, Korea

Received: February 7, 2020 Accepted: February 14, 2020

\section{Correspondence to}

Pil Soo Sung, M.D.

Department of Internal Medicine, Seoul St. Mary's Hospital, College of Medicine, The Catholic University of Korea, 222 Banpo-daero, Seocho-gu, Seoul 06591, Korea

Tel. +82-2-2258-2073

Fax: $+82-2-3481-4025$

E-mail: pssung@catholic.ac.kr https://orcid.org/0000-00025780-9607

\section{See Article on Page 320-330}

Serum alanine aminotransferase (ALT) has been used as a marker of hepatocyte injury for decades; however, the total number of damaged hepatocytes does not always correlate with the ALT level [1]. ALT is a critical enzyme that mediates the transamination reaction between alanine and 2-oxoglutarate to form glutamate and pyruvate [2]. Because ALT is abundantly expressed in the liver and scarcely expressed in other tissues, it has traditionally been used as a marker of liver injury [2]. Currently, two isoenzymes of ALT, which are differentially expressed in various tissues, have been identified $[2,3]$. Specifically, ALT1 is expressed in the cytoplasm and ALT2 expressed in the mitochondria $[2,3]$. After liver injury, the levels of both ALT1 and ALT2 are increased in the serum $[2,3]$. ALT1 is mainly distributed in the intestine, liver and muscle, and ALT2 is mainly distributed in the liver [2].

Chronic liver disease (CLD) is caused by constant tissue destruction and regeneration, which results in fibrosis. Chronic hepatitis B (CHB), chronic hepatitis C, non-alcoholic steatohepatitis (NASH), and alcohol-mediated liver injury are the most common eti- ologies of CLD. All of these diseases cause pathological liver fibrosis and liver cirrhosis $[4,5]$. Liver biopsy has traditionally been regarded as the gold standard for determining the fibrosis grade in patients with CLD. However, it only provides limited information, i.e., represents only a small part of the whole liver, and does not reflect dynamic changes that occur during fibrogenesis $[4,6]$. In addition to technical problems, liver biopsy remains an invasive procedure that can cause potentially life-threatening complications such as bleeding [6]. Due to these limitations, non-invasive methods to evaluate the extent of liver fibrosis are urgently needed. To date, transient elastography, magnetic resonance elastography, and shear wave elastography, as well as parameters such as the non-alcoholic fatty liver disease fibrosis score, fibrosis-4 (FIB4) and aspartate aminotransferase to platelet ratio (APRI), can be used to diagnose advanced fibrosis [4]. Recently, the diagnostic performance of a range of non-invasive tests was assessed in patients with NASH, and satisfactory results were reported in terms of their ability to detect advanced fibrosis [6]. More recent studies have drawn attention to a range of candidate biomarkers for fibrotic diseases, including 
matrix metalloproteinases, DNA methylation markers, and matrix neoepitopes, many of which have shown promise as biomarkers in liquid biopsy samples [7]. However, there is still an unmet need for novel markers that can be tested easily by clinicians and used in daily practice.

Almost all liver cirrhosis patients present with persistently normal ALT levels, despite the fact that ALT levels are elevated in hepatocyte injury. The results of a recent study showed that advanced fibrosis was present in approximately $8 \%$, and cirrhosis in up to $6 \%$, of CHB patients with normal ALT levels [8]. Currently, serum ALT levels are measured in clinics according to the catalytic activity of the enzyme [1-3,9]; therefore, the results may not actually represent the total amount of ALT in serum. Immune-mediated liver injury caused by $\mathrm{T}$ cells, natural killer cells, and macrophages is critical in the progression of liver fibrosis, and previous studies have reported large areas of immune cell infiltration in livers with advanced fibrosis; as such, serum ALT levels measured using enzymatic methods may be normal $[10,11]$.

In this issue of the Korean Journal of Internal Medicine, Kim et al. [9] investigated the efficacy of enzyme-linked immunosorbent assay (ELISA) to detect ALT isoenzymes for predicting liver fibrosis and inflammation, and demonstrated significant correlations of ALT1 levels with inflammation grade and fibrosis stage. Currently, enzymatic assays of ALT are typically used to determine serum levels of the protein [12]. However, enzymatic assays cannot accurately detect liver injury when the fibrotic burden is severe [12]. A previous report demonstrated that ALT immunoassays, which measure the actual ALT mass concentration, showed higher sensitivity and specificity for liver cirrhosis and hepatocellular carcinoma [12]. In that report, the authors postulated that complex formation between ALT protein and its antibody is more likely in cases of more severe liver disease $[9,12]$. ALT proteins bound to their autoantibodies showing reduced enzymatic function have been identified in patients with CLD; therefore, an assay that accurately measures the concentration of serum ALT is needed [9,12,13].

ELISA is a sensitive tool used for the detection and quantification of specific molecules in sera or culture supernatant. In the field of laboratory-based medicine,
ELISA has contributed greatly to the detection of disease-specific molecules. Immunological methods such as immunoblotting and flow cytometry, as well as ELISA, have enabled researchers and clinicians to accurately quantify target molecules. In the manuscript of Kim et al. [9], Fig. 3B shows that the ALT1 mass concentration, obtained using sandwich ELISA, was significantly associated with fibrosis stage and inflammation grade in CHB patients. On the other hand, ALT values measured using enzymatic activity did not reflect the degree of fibrosis.

Overall, the findings reported by Kim et al. [9] suggest that ALT1 detection using an immunologic assay could be valuable for diagnosing fibrosis progression in patients with CLD. These valuable results suggest that ALT1, which can be measured non-invasively by immunoassay, is an "easy to measure" marker for liver fibrosis. Readers should also note that by using a different detection method, a commonly analyzed serum marker was identified as an indicator of a chronic pathologic condition.

\section{Conflict of interest}

No potential conflict of interest relevant to this article was reported.

\section{Acknowledgments}

This research was supported by the Basic Science Research Program through the National Research Foundation of Korea (NRF) funded by the Ministry of Education (NRF-2019R1I1A1A01059642).

\section{REFERENCES}

1. Sookoian S, Pirola CJ. Liver enzymes, metabolomics and genome-wide association studies: from systems biology to the personalized medicine. World J Gastroenterol 2015;21:711-725.

2. Yang RZ, Park S, Reagan WJ, et al. Alanine aminotransferase isoenzymes: molecular cloning and quantitative analysis of tissue expression in rats and serum elevation in liver toxicity. Hepatology 2009;49:598-607.

3. McAllister CH, Facette M, Holt A, Good AG. Analysis of the enzymatic properties of a broad family of alanine aminotransferases. PLoS One 2013;8:e55032. 
4. European Association for Study of Liver; Asociacion Latinoamericana para el Estudio del Higado. EASL-ALEH clinical practice guidelines: non-invasive tests for evaluation of liver disease severity and prognosis. J Hepatol 2015;63:237-264.

5. Lo RC, Kim H. Histopathological evaluation of liver fibrosis and cirrhosis regression. Clin Mol Hepatol 2017;23:302-307.

6. Anstee QM, Lawitz EJ, Alkhouri N, et al. Noninvasive tests accurately identify advanced fibrosis due to NASH: baseline data from the STELLAR trials. Hepatology 2019;70:1521-1530.

7. Tatler AL. Recent advances in the non-invasive assessment of fibrosis using biomarkers. Curr Opin Pharmacol 2019;49:110-115.

8. Cristina SJL, Marta CM, Mercedes GS, et al. Characterization and evaluation of liver fibrosis grade in patients with chronic hepatitis B virus infection and normal transami- nases. Clin Mol Hepatol 2018;24:384-391.

9. Kim HJ, Kim SY, Shin SP, et al. Immunological measurement of aspartate/alanine aminotransferase in predicting liver fibrosis and inflammation. Korean J Intern Med 2020;35:320-330.

10. Sutti S, Albano E. Adaptive immunity: an emerging player in the progression of NAFLD. Nat Rev Gastroenterol Hepatol 2020;17:81-92.

11. Van Herck MA, Weyler J, Kwanten WJ, et al. The differential roles of $t$ cells in non-alcoholic fatty liver disease and obesity. Front Immunol 2019;10:82.

12. Kim HJ, Oh SW, Kim DJ, Choi EY. Abundance of immunologically active alanine aminotransferase in sera of liver cirrhosis and hepatocellular carcinoma patients. Clin Chem 2009;55:1022-1025.

13. Maekawa M, Abe H, Hara K. A novel explanation for low alanine aminotransferase activity in human serum by IgG inhibitor. Clin Chem Lab Med 2002;40:848-849. 